\title{
ANALYSIS OF DALLARA T12 RACE CAR FRONT WING VIBRATIONS
}

\author{
Pavel Alekseevich Sorokin ${ }^{a}$, Kirill Stanislavovich Khryakov $^{b, *}$, \\ Michiel GOMMERS ${ }^{b}$ \\ ${ }^{a}$ Russian University of Transport (RUT - MIIT), Institute of Transport Engineering and Control Systems, \\ Obrazcova 9b9, 127994 Moscow, Russia \\ ${ }^{b}$ Charouz Racing System SE, Tovární 500, 26753 Žebrák, Czech Republic \\ * corresponding author: kirillkhryakov@gmail.com
}

\begin{abstract}
This work investigates aerodynamic elements' vibrations of high-speed vehicles on the example of the Dallara T12 race car front wing. Made up energy spectrums of wing vibrations for three specific cases arising during driving on the circuit.
\end{abstract}

KEYWORDS: race car; Dallara T12; front wing; sensors; spectral analysis; energy spectrum; vibration frequency.

\section{INTRODUCTION}

Motorsport is the main testing ground for all innovations and technical solutions used in the road transport. Racing, where the difference between winning and losing is a matter of few tenths of a second, encourages the leading manufacturers of racing cars to invest huge amount of resources in the development and improvement of their cars.

In the wide spectrum of all kinds of racing series, the top of the motorsport is rightfully taken by circuit racing. The main reason for the beneficial effect of circuit racing on the development of the road transport is that the main task of the car in this kind of racing is not to reach the maximum record speed, but to pass a complex circuit with different turns in the shortest time.

From this, it follows that the best car for this kind of racing is a car capable of driving equally fast on straight lines of the road and in the corners. This means that the car should have a good acceleration, effective braking, and be able to keep the cornering speed as high as possible.

However, fast driving in corners is limited by the frictional force between the wheels and the asphalt. One of the most effective ways to increase this force is the use of aerodynamic elements generating the downforce, which allows to press the car more tightly to the road [1, 2].

A lot of new aerodynamic elements, which were tested in wind tunnels or developed with CFD modelling (Computational Fluid Dynamics), were designed based on stationary conditions. In actual conditions, when the car is moving on the road, vibrations, which reduce the efficiency of these elements, occur.

It becomes obvious that it is a great practical interest to study the influence of vibrations on the downforce level. To start this research, it is necessary to first investigate the vibrations of aerodynamic elements. In this article, the vibrations of the Dallara T12 racing car's front wing are studied [3, 4].

\section{Preparation FOR EXPERIMENTAL RESEARCH}

The assembly of the Dallara T12 racing car's front wing (Figure 1) consists of carbon parts fastened together by steel bolts [5].

The mainplane of the wing is fixed at the ends with the endplates, which serve to stabilize the airflow along the edges of the wing and eliminate the end vortices. In the rear part of the endplate, there is a nolder, which improves the interaction of the airflows that escape from the wing with the vortex flows formed around the rotating wheel of the car located just behind the front wing [6].

In the middle of each console part of the mainplane of the wing, there are flap mountings. Between these mountings and endplates are installed flaps of the wing, fixed with bolts.

In the endplates and flap mountings, there are 32 holes for changing the angle of attack of the flap. Thus, by moving the adjusting bolt between these holes, it is possible to adjust the level of the downforce of the front wing by changing the angle of attack of the flap [7]. In this case, the minimum angle of attack is $0^{\circ}$, provides the lowest level of downforce and the least aerodynamic drag. The maximum angle of attack of $32^{\circ}$, corresponds to the maximum level of the downforce, but also leads to the maximum value of air drag. Thus, by adjusting the angle of attack of the wing with a step of $1^{\circ}$, you can achieve the best balance in the ratio of downforce - drag, depending on the race track.

The assembled wing, using special mainplane mountings made of an alloy based on aluminium, is 


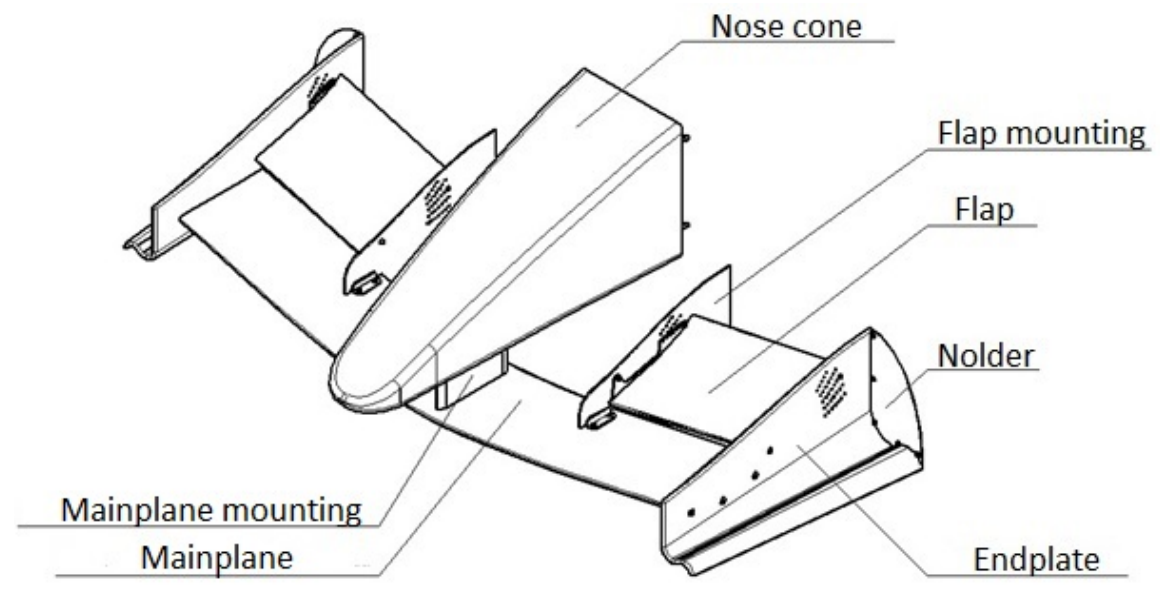

FiguRE 1. The assembly of the Dallara T12 racing car's front wing.

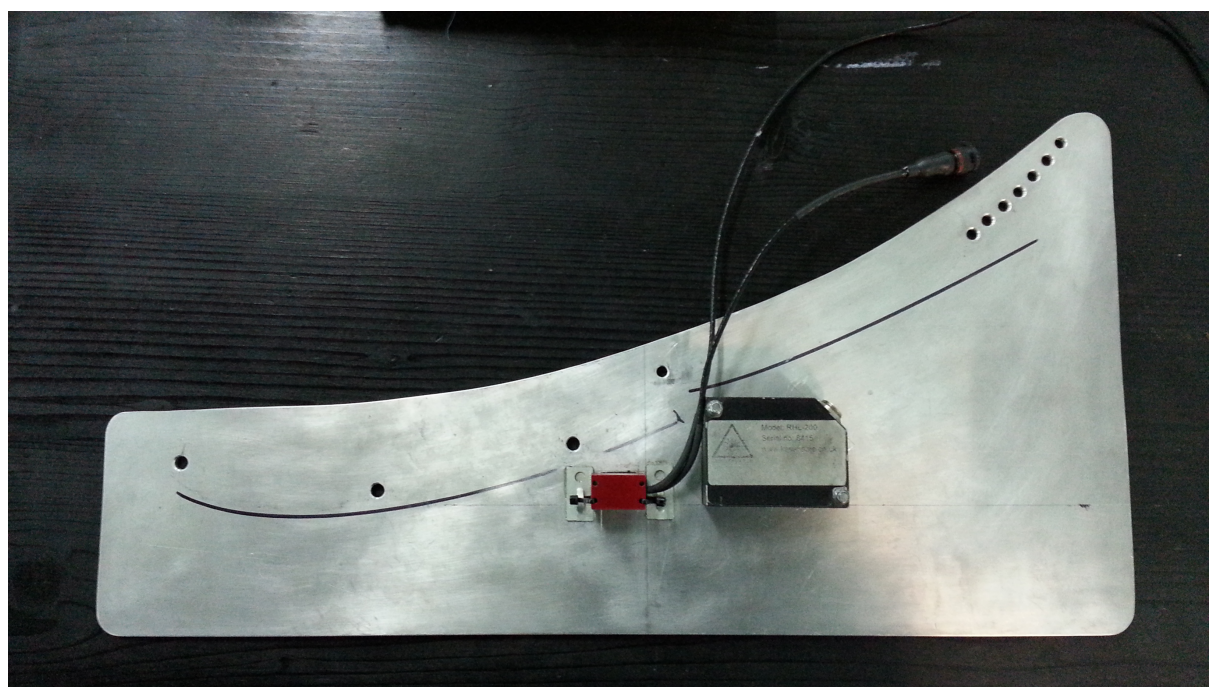

FIGURE 2. Aluminium bracket with mounted sensors for measuring the vibration of the wing.

attached to the nose cone of the car. To improve the aerodynamic flow, these mountings are closed with special carbon fairings. The nose cone assembly with a wing is fixed to the chassis of the car with four quickrelease bolts, providing a reliable and rigid fastening with the possibility of a quick dismantling.

The assembly of the Dallara T12 racing car's front wing, due to its design features, is most prone to vibrations of two types: vertical oscillations of the mainplane of the wing and twisting vibrations of the assembly endplate - flap - flap mounting - mainplane of the wing [8].

To measure the characteristics of these vibrations, it was suggested to install two sensors: one for measuring the vertical displacements, the second for measuring the twisting.

To measure the vertical movements of the wing, a laser sensor K-RHL2, made by the company "CA Sensors" (Great Britain), was used for measuring the ride height (clearance) .

The K-RHL2 sensor operates on a non-contacting principle, measuring the distance to the road with a laser beam. Reflected from the surface of the road, the laser beam is perceived by the CCD-matrix, by means of which the distance to the surface is measured.

The signal from the sensor is transmitted via a 3core cable (power, signal, ground), which is connected to the sensor using the special purpose connector ASL006-05PC-HE, manufactured by Deutsch (USA).

To obtain the characteristics of the twisting movements of the wing, the GYRP-1 axis gyro sensor manufactured by "Texense" (France) was used.

The sensor is made in the form of a MEMS device (microelectromechanical system) and measures the speed of rotation of the sensor body around one axis. Inside the gyro sensors, made on the basis of MEMS device, there is a vibrating silicon ring, which allows to provide a high accuracy of measurement in conditions of strong vibrations. When this ring of angular velocity is acted upon, a Coriolis force arises that deforms the ring and thereby causes its radial displacements.

Along the perimeter of the ring, there are uniformly installed drive-converters. Some of them excite and control the primary working amplitude of the vibration of the gyro sensor and the other transducers deter- 


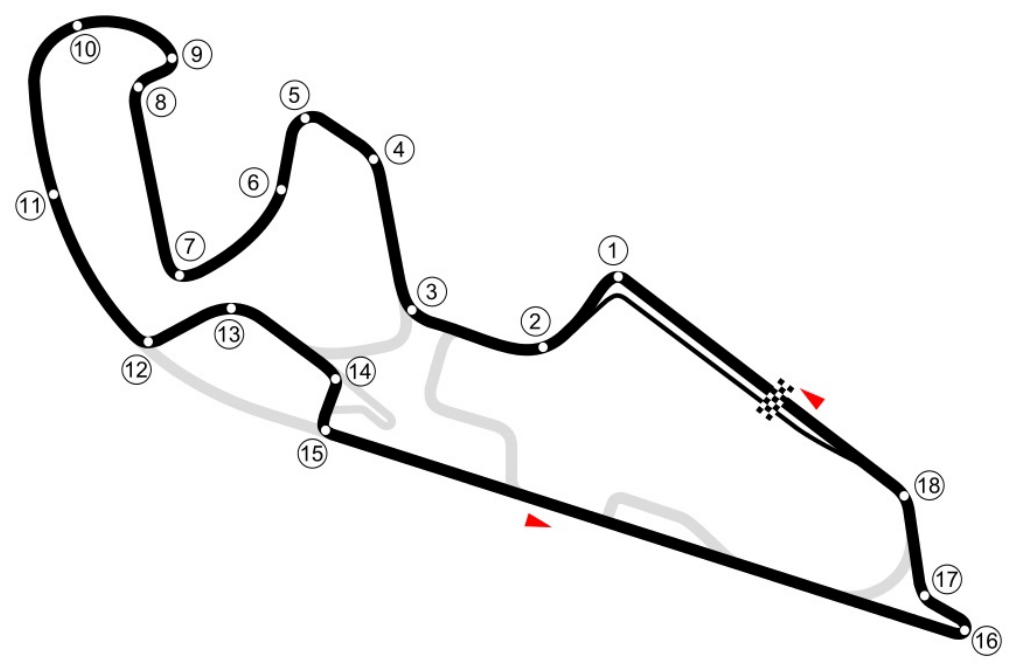

Figure 3. Motorland Aragon race track layout.

mine the radial displacements caused by the Coriolis force and the proportional angular velocity of the rotation. All converters work in pairs. Sensors-gyroscopes working on this principle are placed in a sealed enclosure that allows to keep a vacuum environment inside and thus prevent the influence of changing physical air parameters on the accuracy of the output signal.

The signal from the sensor is transmitted through a 3-wire cable (power, signal, ground) 3X26AWG FEP, which is connected to the central circuit of the car using the special purpose connector ASL606-05PNHE, manufactured by Deutsch (USA).

Both sensors were securely attached to the aluminium bracket shown in Figure 2, The bracket was installed between the mainplane of the wing, the flap and the endplate on the right hand side of the car. This technical solution allowed the sensors to be rigidly fixed on the elements of the wing, while not violating their integrity by drilling additional mounting holes.

Signals from the sensors were transmitted to the vehicle's on-board computer, located in the cockpit of the driver. Sensor cables were laid along the trailing edge of the mainplane of the wing, which ensured the least loss of downforce.

However, due to the fact that the installation of the bracket with sensors and the laying of cables somewhat violated the aerodynamic efficiency of the right part of the wing and partly reduced the downforce, it was decided to increase the angle of attack of the right flap by one position $\left(+1^{\circ}\right)$. This decision was based on the fact that the sensor cables were attached to the wing trailing edge by using the tesa tape. The rough surface of the tape slightly disturbed the boundary layer, which could lead to an earlier separation of the flow from the lower surface of the flap. To compensate for a slight drop in the downforce caused by an earlier flow separation, it was decided to increase the flap attack angle by the minimum possible value ( $1^{\circ}$ only). According to Dallara T12 Aerodynamic manual [9], an increase of the flap attack angle by $2^{\circ}$ will lead to a significant increase of downforce, which can have an effect on the car balance and disturb the driver. Thus, the angle of attack of the left flap was $23^{\circ}$, and the right angle of attack was $24^{\circ}$.

This configuration of the front wing's settings allowed to level the aerodynamic balance of the car and to simplify its management.

With the help of a special software to control the on-board computer of the car, the vibration measurement sensors have been calibrated in an accordance with the recommendations of the manufacturers. The frequency of reading of the signal was set to $100 \mathrm{~Hz}$ for saving the memory of data logger and for a convenience of comparison with other parameters of the vehicle dynamics, measured by sensors with a maximum frequency of $100 \mathrm{~Hz}$. For this type of research, this frequency of signal measurement is sufficient, which will be further confirmed by results of the research.

Also, during the tests we, used standard sensors installed on the car and measured the following parameters: car speed, rpm, longitudinal and lateral acceleration of the car (the sensor is installed near the centre of gravity of the car), steering angle, throttle position, pressure in the brake system, gear change, activation and deactivation of the active rear wing flap (drag reduction system), the movement of the shock absorbers, roll on the front and rear axles of the car.

The analysis of the above parameters allows us to determine the movements and vibrations of the chassis of the car, which must be taken into account when determining the relative vibrations of the front wing.

\section{EXPERIMENTAL RESEARCH}

Experimental research to determine the characteristics of the vibration of the Dallara T12 racing car's front wing were conducted on the Motorland Aragon race track (Spain). A layout of this track is presented in Figure 3 . 


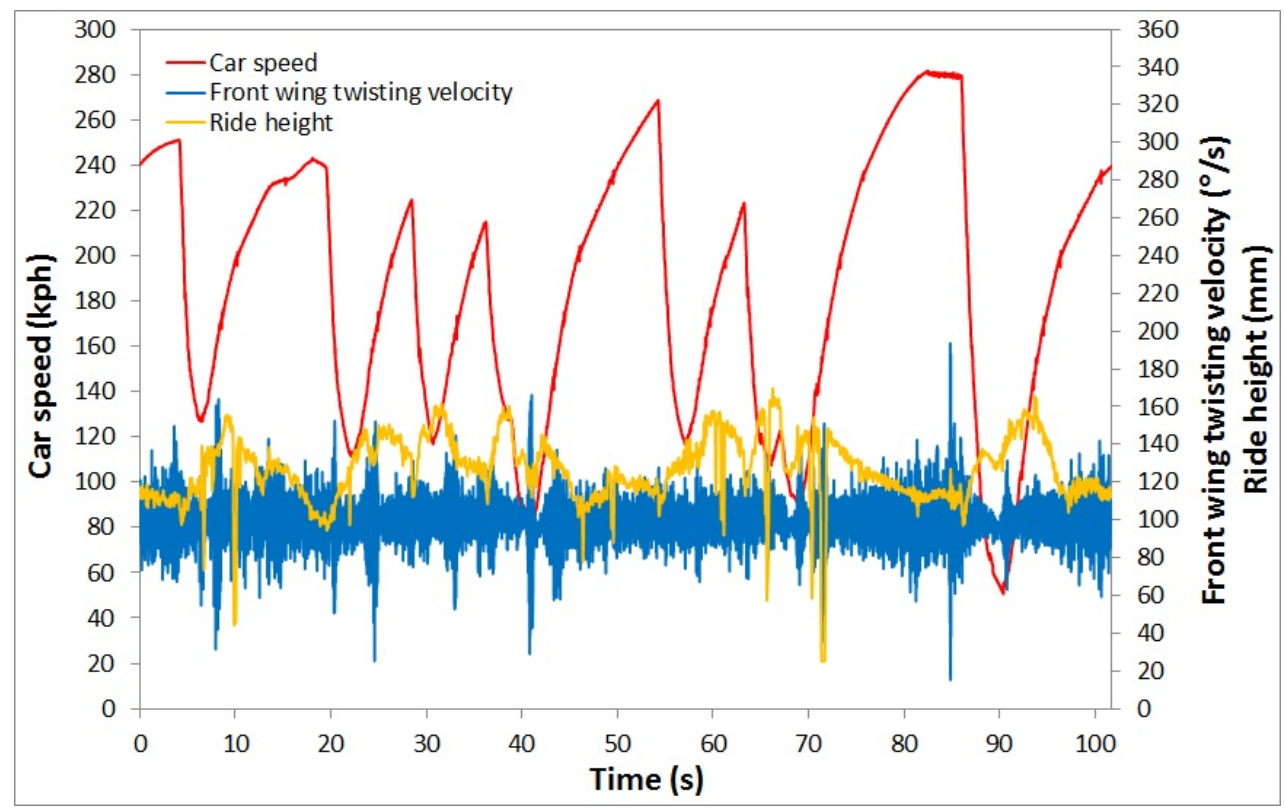

FiguRE 4. Dallara T12 racing car's front wing vibration graph.

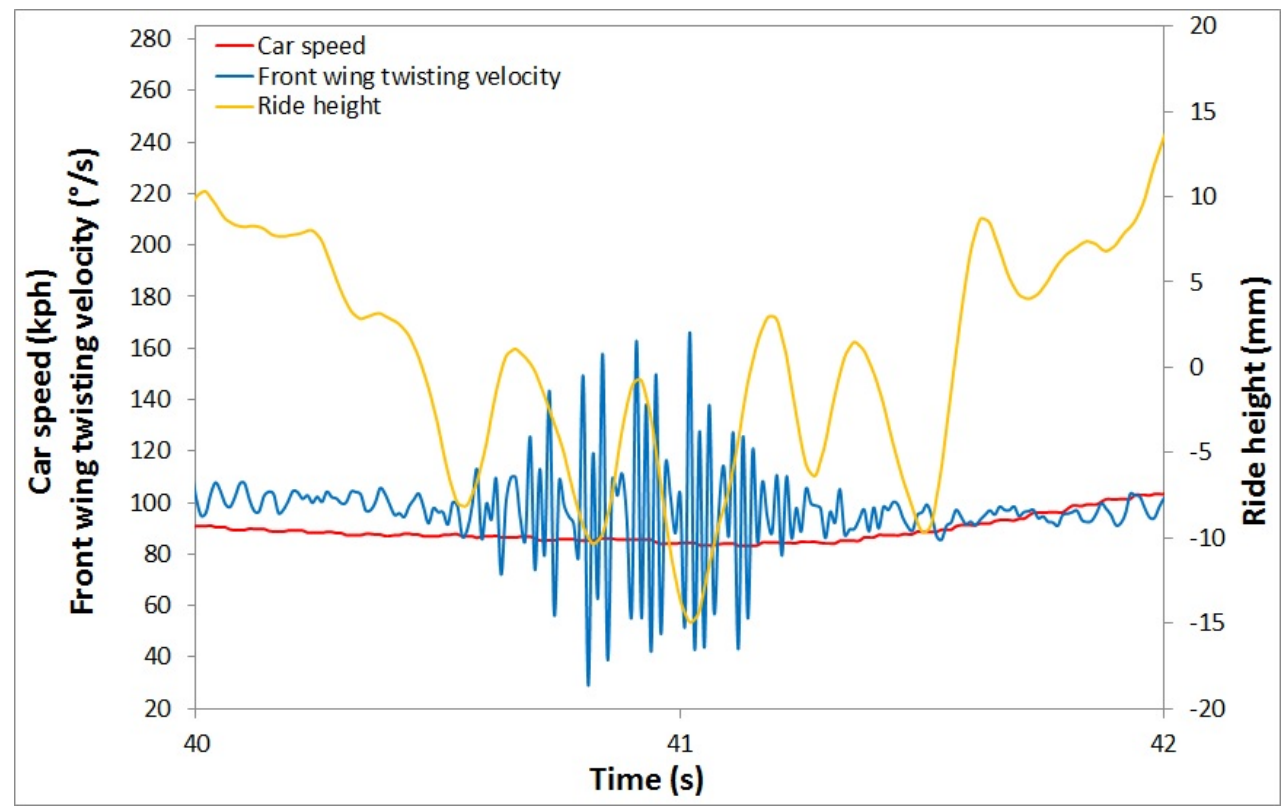

FIgURE 5. Front wing vibration graph in the first characteristic case.

The configuration of the track, $5345 \mathrm{~m}$ long, contains 18 turns with different radii. The maximum elevation difference is $50 \mathrm{~m}$.

The driver passed 8 laps, which totals to $42.239 \mathrm{~km}$, and showed a fastest lap time of $1: 40.730$ (the time is represented in the format minutes:seconds.thousandths of a second).

During the test runs, the southeast wind blew at a speed of $5 \mathrm{~m} / \mathrm{s}$. The air temperature was $13.3^{\circ} \mathrm{C}$, the temperature of the asphalt was $16{ }^{\circ} \mathrm{C}$, and the air humidity was about $55 \%$.

The data obtained as a result of testing, for the convenience of perception, are expediently presented in the form of a graph in which the time is measured along the abscissa axis and the physical quantities (speed, displacements, etc.) are measured along the ordinate axis.

In Figure 4 there is a graph showing the car speed in conjunction with the vertical and twisting deformations of the wing during the fastest lap.

This form of representation of experimental data makes it quite easy to determine where the car was located at a certain point in time. The smooth growth of the car's speed (red line) corresponds to the movement along the straight section of the track with acceleration and the sudden drop in speed corresponds to braking before the sharp turns.

Figure 4 clearly shows that the oscillations are a non-stationary process, depending on a variety of different factors. These include: speed, acceleration, 


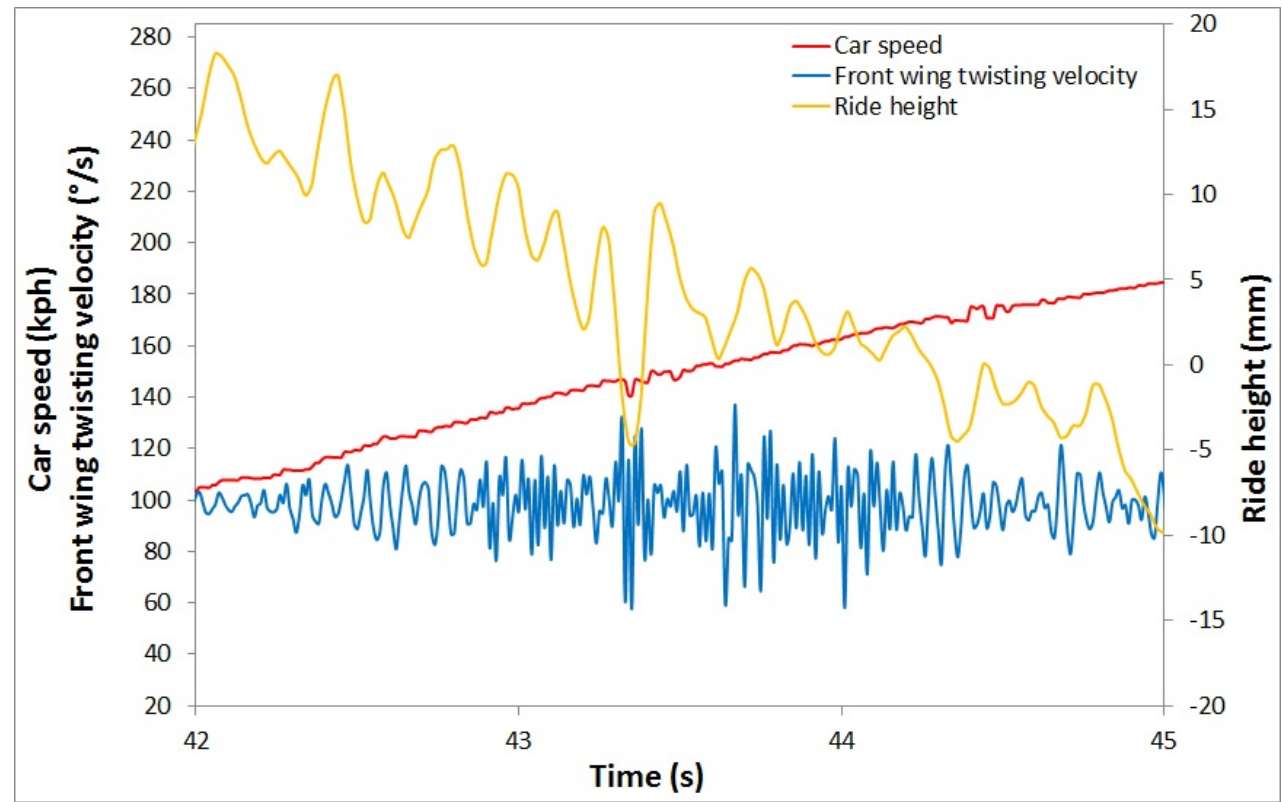

FiguRE 6. Front wing vibration graph in the second characteristic case.

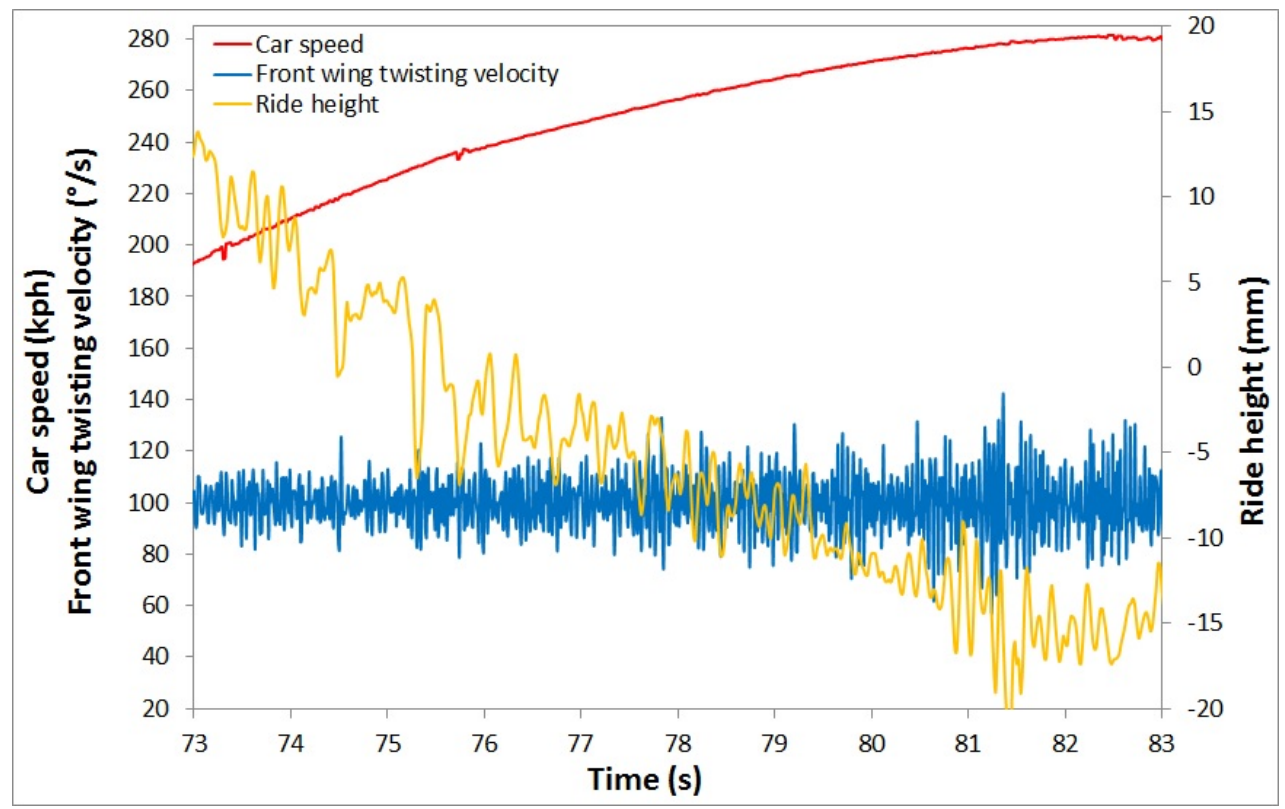

FiguRE 7. Front wing vibration graph in the third characteristic case.

curvature of the turn, the quality of the road, driving style of the driver, car set-up, etc.

However, a practical interest is the behaviour of the wing in specific situations, for example, at the entrance to the turn, when exiting from it, while driving along a straight section of the road, etc. Therefore, it is advisable to identify the "characteristic" areas on the graph and subsequently explore them separately, as processes independent of each other.

The first characteristic case corresponds to a collision on a high curb at the entrance to Turn 9 . The car drives at a constant speed of $85 \mathrm{~km} / \mathrm{h}$ with left wheels on a curb height of $4 \mathrm{~cm}$ (Figure 5).

The second characteristic case corresponds to the collision on the low curb at the exit from Turn 9. The car accelerated from 103 to $185 \mathrm{~km} / \mathrm{h}$ in 3 seconds, with right wheels driving on a curb height of $2 \mathrm{~cm}$ (Figure 6).

The third characteristic case corresponds to driving along a straight section of the track between Turns 15 and 16. The car accelerated from 193 to $280 \mathrm{~km} / \mathrm{h}$ in 10 seconds, moving along a straight trajectory without entering the curbs (Figure 7).

\section{SPECTRAL ANALYSiS \\ OF AN EXPERIMENTAL DATA}

Due to the random nature of the front wing vibrations, the best way to analyse it is using the spectral analysis method [10]. 


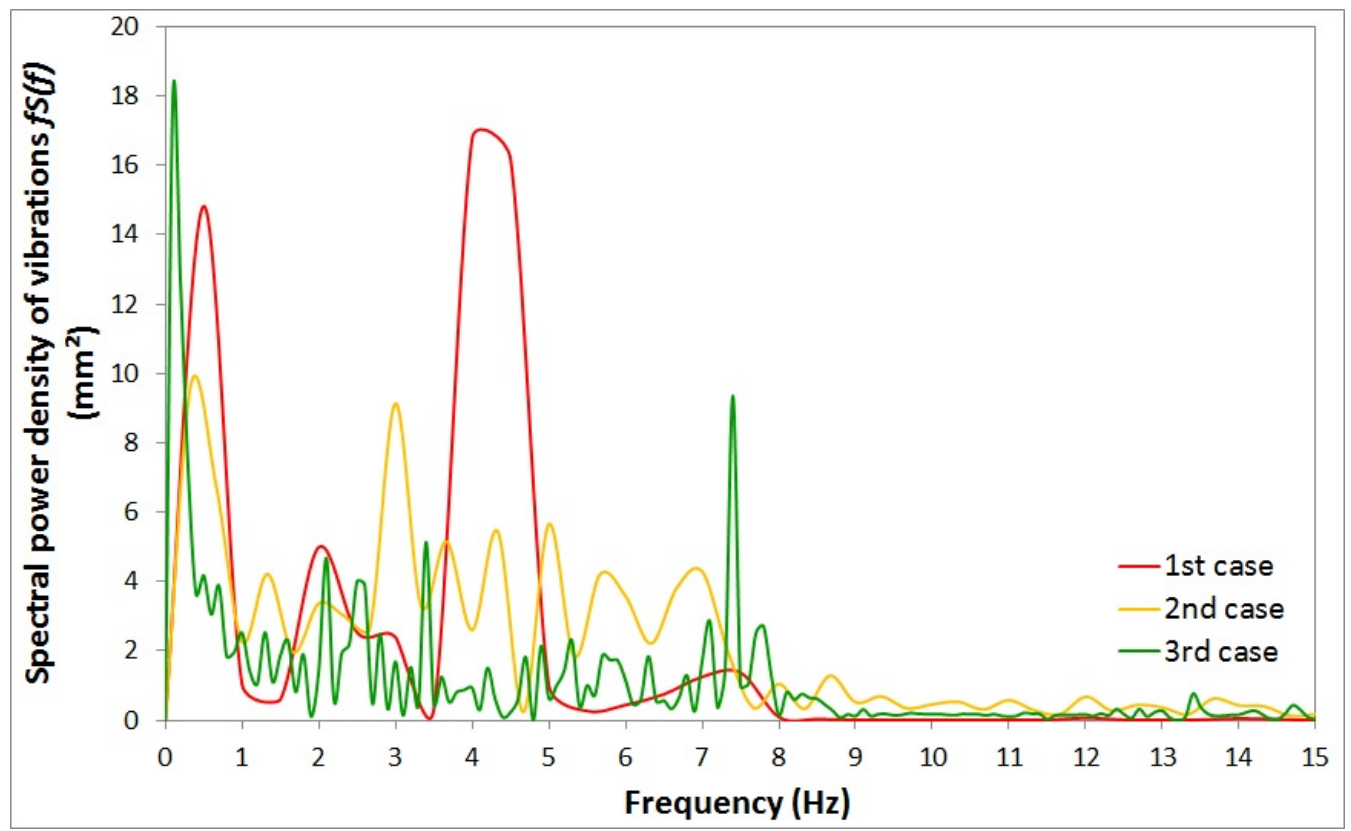

Figure 8. Spectral power density of front wing vibrations $f S(f)$.

Spectral analysis allows representing the energy distribution of oscillations in frequencies. The frequency intervals with the highest energy indicate that the oscillations with these frequencies occur most often, or they have the greatest amplitude [10, 11].

The values of the vertical movements of the wing for each characteristic case are expediently represented in the form of energy spectra that distribute the energy of these oscillations in the frequency domain.

These energy spectra, also called the spectral power density of the oscillations, will make it possible to clearly determine the frequency with which the wing oscillations occur most frequently, or they have the largest amplitudes.

Calculation of the spectral power density of oscillations was performed using the calculation module "sptool" of the MATLAB software [12, 13.

The energy spectrum of the oscillations was constructed by the fast Fourier transform (FFT) method. Spectral power density graphs of the Dallara T12 racing car's front wing vibrations for all three characteristic cases are presented in Figure 8.

To display the spectral power density of the wing vibration $S(f)$ on a scale convenient for a visual perception, each value of the spectrum was multiplied by the corresponding frequency $f$. Thus, in Figure 8 shows the spectrum of $f S(f)$.

Analysing the spectrum, we can conclude that the oscillations with frequencies close to 1 and $4 \mathrm{~Hz}$ have the largest energy in the first characteristic case. Also, a significant part of the oscillation energy is located in the region of 2 and $7 \mathrm{~Hz}$. The distribution of energy over these frequencies is due to low-frequency oscillations, with large amplitudes resulting from driving on a high curb at low speed in the first characteristic case.
In the second characteristic case, the oscillations with frequencies close to 1 and $3 \mathrm{~Hz}$ have the greatest energy. However, in contrast to the first characteristic case, in this spectrum there are no clearly distinguished frequencies with a high oscillation energy. A significant amount of energy is concentrated in the frequency range from 0 to $7 \mathrm{~Hz}$. Such a distribution of energy over frequencies is due to a higher speed of the car in comparison with the first characteristic case as well as a low curb height.

In the third characteristic case, the highest energy level has oscillations with frequencies close to $2.5 \mathrm{~Hz}$. Also, a large amount of vibration energy is concentrated in regions 1 and $7.5 \mathrm{~Hz}$. The remaining energy is distributed in the range from 0 to $20 \mathrm{~Hz}$. Driving with a high speed along the straight sections of the race track leads to a decrease in the amplitude of the oscillations and an increase in their frequency. The energy of the oscillations is uniformly distributed throughout the frequency domain, with the exception of isolated peaks. This trend is manifested due to increased high-frequency vibrations of the engine and transmission as well as the occurrence of autooscillations of the wheels caused by errors in their balancing [14.

To estimate the probability of the resonance of the Dallara T12 front wing, a modal analysis of its finite element model was performed. The frequencies of the first ten forms of its own oscillations were determined. The lowest natural frequency corresponding to the first mode of oscillation was $216 \mathrm{~Hz}$, which significantly exceeds the frequencies obtained as a result of spectral analysis of experimental studies. In this regard, we can conclude that on the front wing of Dallara T12 race car, the occurrence of resonance is unlikely. 


\section{Conclusions}

The data obtained as a result of the spectral analysis of three characteristic cases of the Dallara T12 racing car's front wing vibrations give a clear idea of the characteristics of the wing oscillations while driving the car along the track.

The use of these results in carrying out the CFD analysis or blowing in the wind tunnel as the initial data for the formation of vibrational disturbances will allow investigating the effect of vibrations of the race car aerodynamic elements on the level of the downforce.

The results of the modal analysis of the finite element model of the Dallara T12 racing car's front wing shows that lowest natural frequency of the wing significantly exceeds the frequencies obtained as a result of spectral analysis of experimental studies. In this regard, we can conclude that an occurrence of a resonance is unlikely for this wing. However, when designing new racing cars, it is advisable to check a possibility of a resonance of all aerodynamic elements, which generate downforce.

\section{REFERENCES}

[1] J. A. Dominy, R. G. Dominy, "Aerodynamic influence on the performance of the Grand Prix racing car", Proceeding of the institution of mechanical engineers, no. 7 , pp. $87-93,1984$.

[2] J. R. Jagadeep, G. Mayank, "Finding the optimum angle of attack for the front wing of an F1 car using CFD", Proceedings of the 4th WSEAS International Conference on Fluid Mechanics and Aerodynamics, pp. 29 - 34, Greece, 2006.
[3] V. D. Berg, "Aerodynamic interaction of an inverted wing with a rotating wheel", Southampton, 2007.

[4] K. S. Khryakov, "Problems of the efficient operation of race car aerodynamic elements", Tidings of the Tula State University. Technical sciences, no.11, pp. $193-$ 196, Russia, 2014.

[5] T12 2012 spare parts catalog, Dallara automobili, 2011.

[6] M. Amadori, "Design and development of the new composite-material mainplane of the Dallara T12 race car", Bologna, 2013.

[7] J. Katz, "Race car dynamics: designing for speed", Cambridge: Bentley Publishers, 1995.

[8] K. S. Khryakov, P. A. Sorokin, "Research of the shapes and natural frequencies of the Dallara T12 race car front wing", Tidings of the Tula State University. Technical sciences, no.5, pp. 85 - 91, Russia, 2015.

[9] T12 2012 aerobook, Dallara automobili, 2012.

[10] A. A. Afonsky , V. P. Dyakonov, "Digital analyzers of spectrum, signals and logic", SOLON-PRESS, 2009.

[11] A. B. Sergienko, "Digital signal processing", BHV-Petersburg, 2011.

[12] K. Chen, A. Giblin, A. Irving, "Matlab in Mathematical Research", 2001.

[13] Yu. Lazarev, "Modeling of processes and systems in MATLAB. Training course", Publishing Group BHV, 2005.

[14] W.F. Milliken, "Race car vehicle dynamics", Warrendale: SAE International, 1995. 